\title{
Radiotherapy during COVID-19 pandemic. How to create a No fly zone: a Northern Italy experience
}

\author{
Giampaolo Montesi ${ }^{1}$. Saide Di Biase ${ }^{1}$. Sara Chierchini ${ }^{1}$. Giovanni Pavanato ${ }^{1}$. Graziella Elia Virdis ${ }^{1}$. \\ Edgardo Contato ${ }^{2} \cdot$ Giovanni Mandoliti $^{1}$
}

Received: 8 April 2020 / Accepted: 27 April 2020 / Published online: 15 May 2020

(c) Italian Society of Medical Radiology 2020

\begin{abstract}
Background SARS-CoV-2 pandemic represents a troubling health emergency but also a main challenge for the clinical governance of the system. Discontinuation of radiation treatments is not desirable and potentially life-threatening. On the other hand, accesses to hospital expose cancer patients to an increased risk of COVID-19 infection. We report our extended protocol, draft to manage clinical activities in our radiotherapy department, by minimizing contagion risks.

Methods We used telephonic screening to assess the need for patient admission. A telephonic triage was performed to identify the presence of COVID-19 infection risk factors or symptoms. New treatments were stratified according to priority codes. A reserved entrance to radiotherapy department was assured for patients and staff. Surgical disposable mask was required for patients and caregivers. The activities were distributed during the whole workday, avoiding overlap to reduce aggregation. Results From 1st February 2020 to 31 March 2020, we reported an increase in the number of first medical examinations and treatments, compared to the same period of the previous year. Outpatients first medical examinations have been spread over the 12 working hours. No COVID-19 cases were detected.

Conclusion During COVID-19 pandemic, we introduced procedures that allowed us to ensure the continuity in oncological cares, with limited risks of infection for patients and staff.
\end{abstract}

Keywords COVID-19 $\cdot$ Pandemic $\cdot$ SARS-CoV-2 $\cdot$ Coronavirus $\cdot$ Radiotherapy $\cdot$ Radiation oncology

Giampaolo Montesi

giampaolomontesi@gmail.com

Saide Di Biase

saide.dibiase@aulss5.veneto.it

Sara Chierchini

sarachierchini@gmail.com

Giovanni Pavanato

giovanni.pavanato@aulss5.veneto.it

Graziella Elia Virdis

graziella.virdis@aulss5.veneto.it

Edgardo Contato

edgardo.contato@aulss5.veneto.it

Giovanni Mandoliti

giovanni.mandoliti@aulss5.veneto.it

1 Radiation Oncology Unit, Radiotherapy Department, Santa Maria della Misericordia Hospital, ULSS5, Rovigo, Italy

2 General Administrative Office, Santa Maria della Misericordia Hospital, Rovigo, Italy

\section{Introduction}

Since SARS-CoV-2 spread throughout the world, clinicians operating out of urgency area, faced out an overwhelming issue concerning the clinical governance of the system.

In high-risk areas, directions of hospitals, closed outpatient treatments and follow-up, except for oncological ones. As a result, radiation oncology services have been called to such an effort: to limit virus breakout, keeping life-saving treatments delivery $[1,2]$.

From that point on, specific directives for radiation therapy units have been lacking, thus we promptly developed some containment procedures, in order to bound virus propagation. 


\section{Methods}

\section{Assess the need for admission: Telephonic screening}

In our experience, the day before follow-up visits, radiation oncologists contact by phone scheduled patients. The interview is aimed to assess general health condition, the presence of neoplasm-related symptoms, the results of prescribed tests and/or reports of diagnostic radiology exams.

In case of stable or not evident disease, the appointment is cancelled and rescheduled as soon as possible. Dedicated additional follow-up workdays have been instituted.

In the eventuality of disease progression or clinical uncertainty the appointment is confirmed and the telephonic triage is performed (ref. Telephonic triage).

\section{Patients who need access to radiotherapy department: Telephonic triage}

All patients planned to access in the radiotherapy department (radiation treatment, simulation CT, non-deferrable follow-up visits and first medical examinations) are contacted by radiotherapy nurses the day before the scheduled appointment. The patients are asked about the presence of fever, cough, flu-like symptoms (arthralgia, myalgia, cold, diarrhoea), about the contact with positive or suspect case of COVID-19 and finally about the geographical provenience. If no risk factors are reported, the patient is admitted in the department accompanied by maximum one caregiver. The patients and caregivers are invited to wear a surgical disposable mask. In case that the triage is suspect for symptoms or risk of contagion, the referring physician activates the procedure by contacting the patient general practitioner (GP) or a national dedicate emergence telephone number (ETN).

\section{Patients who need access to radiotherapy department: Reserved entrance}

In order to reduce contacts and infections, radiotherapy staff and patients are invited to access to the department using this gateway rather than the main entrance of the hospital. The patients are instructed by phone about the alternative suggested path.

\section{Select patients who need radiotherapy}

\section{Urgent treatments}

In urgent situations i.e. spinal cord compression, superior vena cava syndrome, life-threatening lower airway obstruction, digestive or respiratory haemorrhage and life-threatening brain lesion, radiation therapy might be delivered within $24-48 \mathrm{~h}$. Therefore, we considered crucial to pre-identify risk classes of potential to contagion by telephonic triage: not-suspicious asymptomatic (low risk) who can be admitted and treated without restrictions and suspicious asymptomatic or suspicious symptomatic (high risk), which should be referred to GP or to ETN, and even sent to diagnostic swab, when recommended [3, 4].

For other palliative non-urgent treatments, i.e. painful metastatic bone lesions, lung cancer causing chest pain or pancoast syndrome, tumours causing nerve root and soft tissue infiltration, relief of impending airways or bowel obstruction, it is generally indicated that radiotherapy should be started within 7 days. In high-risk contact, adequate personal protective equipment (PPE) was required to safely undergo treatments $[5,6]$, while in low-risk class, no specific restriction has been indicated.

\section{Non-urgent treatments}

In non-urgent cases: prostate cancer patients, breast cancer patients, benign CNS tumour or even non-oncologic patients, treatment could be postponed following priority codes that mainly indicates up to 3 months interval from diagnosis to treatment. For elective priority treatments, i.e. head and neck cancers, rectal and anal cancer, gastroesophageal junction, we select patients to avoid delays in consultation and treatment which may adversely affect potentially curable cancer patients [7].

\section{COVID-19 positive patients}

Finally, in COVID-19 positive patients, it is nowadays very difficult to give an indication to settle the risk of cancer complications/risk of infection ratio. Moreover, a potential spread of contagion through health care operators may awfully affect the ability to provide care, also leading to service closure.

Documents and position paper globally tend to advise against the beginning of a radiation treatment in COVID-19 positive patients [8].

\section{Modulation of the activities}

With the aim of reducing the number of people in the waiting-room, all the activities (first medical examination, simulation CT, day hospital access and follow-up visits) are distributed during the whole workday, avoiding overlap. The patients in treatment are located in a second distinct waiting room. Chairs of the two waiting room are spaced with the aim to maintain a minimum distance of $1 \mathrm{~m}$. All patients are recommended to strictly observe the assigned schedule to avoid extra waiting time. 


\section{Results}

From 1st February 2020 to 31 March 2020, we reported an increase in the number of first medical examinations, from 114 to 124 compared to the same period of the previous year. Treated patients raised from 115 to 130 . Outpatients first medical examinations have been spread over the 12 working

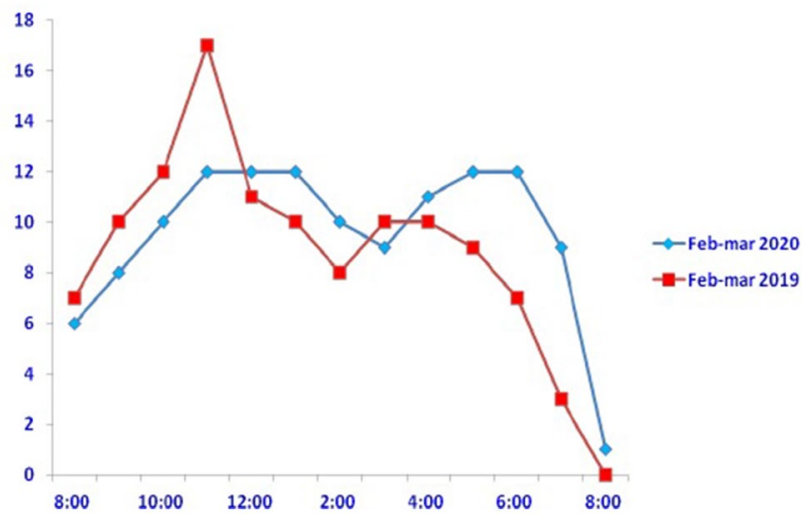

Fig. 1 Timing of consultations over a $12 \mathrm{~h}$ shift during two normal months (red) versus two pandemic months (blue) hours, unlike the previous year, where there was a peak in the middle of the morning (Fig. 1).

In this time lapse, we do not report differences as compared to the same period of 2019 in terms of staff members absence from work (overall 5 days in 2020 vs. 3 days in 2019).

\section{Discussion}

During the spread of the virus, radiotherapy immediately appeared to be marginal in a mass infection setting, but soon returned to play a fundamental role for the maintenance of government-recommended care and to manage many hospital incoming patients [9]. We must underline that data pertained to the entire months of February and March whereas pandemic out-broke since mid-February, thus, the slight increase in the number of patients could be affected by the first 14 days of February.

Our internal protocol (Fig. 2) has permitted to avoid any COVID-19 infection throughout patients and health care professionals, ensuring treatments to our patients along last 2 months, in a region accounting for the $9 \%$ of total infected in Italy $[10,11]$.

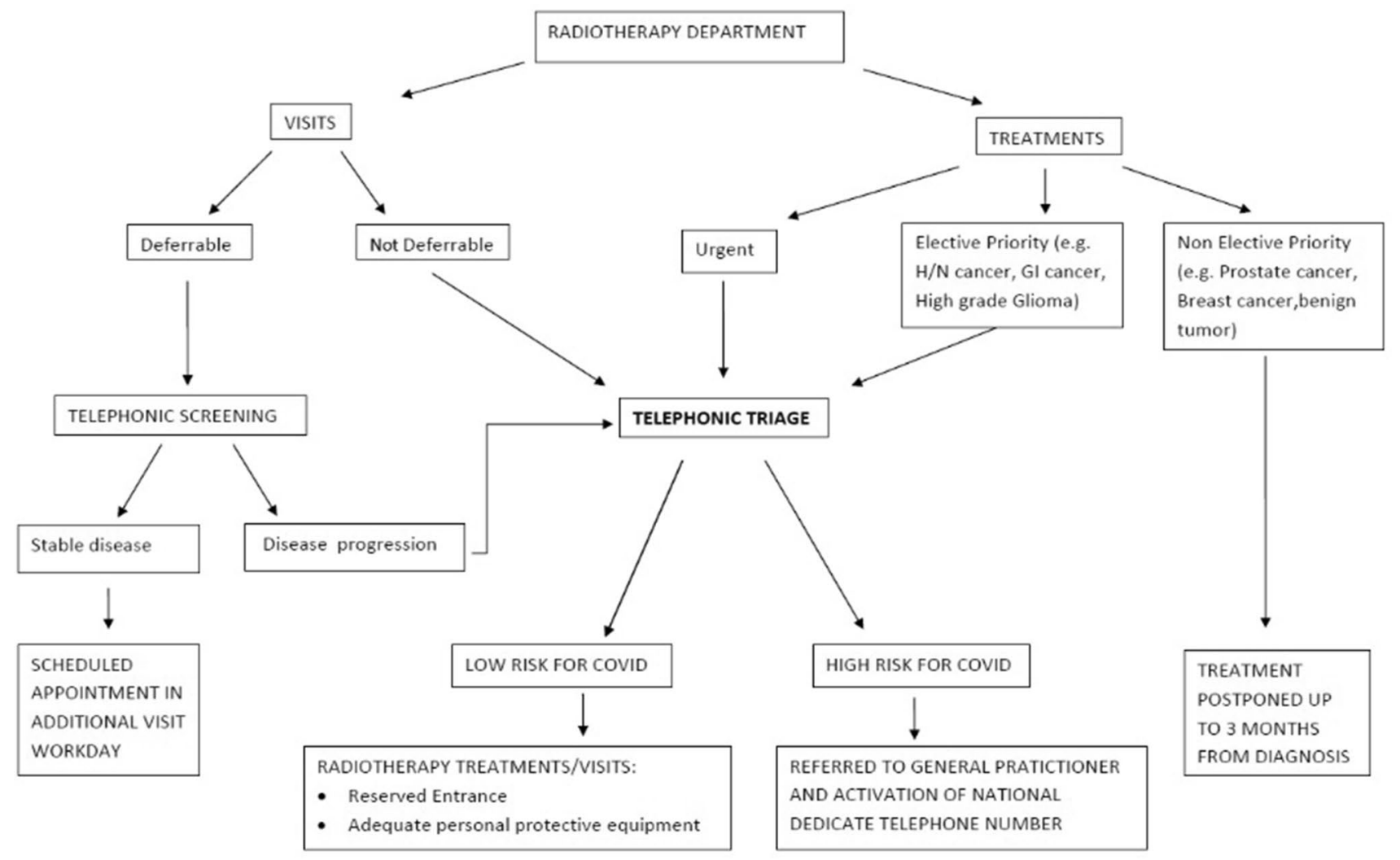

Fig. 2 Protocol flowchart 


\section{Conclusion}

Hoping it will be useful, we reported our original experience, started from the first day of COVID-19 breakout, which allowed us to ensure the continuity of cares, with limited risks.

\section{Compliance with ethical standards}

Conflict of interest The authors declare that they have no conflict of interest.

Ethical standards This article does not contain any studies with human participants or animals performed by any of the authors.

\section{References}

1. Xia Y, Jin R, Zhao J, Li W, Shen H (2020) Risk of COVID-19 for patients with cancer. Lancet Oncol 21(4):e180. https://doi. org/10.1016/S1470-2045(20)30150-9

2. DPCM 01/03/2020 GU Serie Generale n.52 del 01-03-2020

3. Brown S, Kirkbride P, Marshall E (2015) Radiotherapy in the acute medical setting. Clin Med 15(4):382-387. https://doi. org/10.7861/clinmedicine.15-4-382

4. World Health Organization (2020) Laboratory testing for coronavirus disease 2019 (COVID-19) in suspected human cases: interim guidance. World Health Organization. https://apps.who. int/iris/handle/10665/331329. Accessed 2 Mar 2020
5. ECDC Technical Report: Personal protective equipment (PPE) needs in healthcare settings for the care of patients with suspected or confirmed novel coronavirus (2019-nCoV). February 2020

6. Wong J, Goh QY, Tan Z, Lie SA, Tay YC, Ng SY, Soh CR (2020) Preparing for a COVID-19 pandemic: a review of operating room outbreak response measures in a large tertiary hospital in Singapore. Can J Anaesth. https://doi.org/10.1007/s12630-020-01620-9

7. Liang H, Xiang YQ, Lv X, Xie CQ, Cao SM et al (2017) Survival impact of waiting time for radical radiotherapy in nasopharyngeal carcinoma: a large institution-based cohort study from an endemic area. Eur J Cancer 73:48-60. https://doi.org/10.1016/j. ejca.2016.12.009

8. Radiotherapy in a time of crisis, ESTRO Presidents' statement (2020). https://www.estro.org/About/Newsroom/News/Radiothera py-in-a-time-of-crisis

9. Lazzerini M, Putoto G (2020) COVID-19 in Italy: momentous decisions and many uncertainties. Lancet Glob Health. https:// doi.org/10.1016/S2214-109X(20)30110-8

10. WHO (2020) Coronavirus disease (COVID-2019) situation report 56. https://www.who.int/docs/default-source/coronaviruse/situa tion-reports/20200316-sitrep-56-covid-19.pdf?sfvrsn=9fda7 db2_6. Accessed 16 Mar 2020

11. Rubino S, Kelvin N, Bermejo-Martin JF, Kelvin D (2020) As COVID-19 cases, deaths and fatality rates surge in Italy, underlying causes require investigation. J Infect Dev Ctries 14(3):265267. https://doi.org/10.3855/jidc. 12734

Publisher's Note Springer Nature remains neutral with regard to jurisdictional claims in published maps and institutional affiliations. 